\title{
IncRNA PSMA3-AS1 promotes the progression of non-small cell lung cancer through targeting miR-17-5p/PD-L1
}

\author{
Guohua Cheng ${ }^{B-D}$, Yarong LiB , Zhaoyu Liu ${ }^{C}$, Xiang Song ${ }^{A, E, F}$ \\ Department of Oncology, Second Hospital of Shanxi Medical University, Taiyuan, China \\ A - research concept and design; $B$ - collection and/or assembly of data; $C$ - data analysis and interpretation; \\ $D$ - writing the article; $E$ - critical revision of the article; $F$ - final approval of the article
}

Address for correspondence

Xiang Song

E-mail: songxiang96@126.com

Funding sources

None declared

Conflict of interest

None declared

Received on December 7, 2020

Reviewed on May 3, 2021

Accepted on June 7, 2021

Published online on October 5, 2021

Cite as

Cheng $G$, Li Y, Liu Z, Song X. IncRNA PSMA3-AS1

promotes the progression of non-small cell lung cancer

through targeting miR-17-5p/PD-L1. Adv Clin Exp Med.

2021;30(10):1043-1050. doi:10.17219/acem/138624

DOI

10.17219/acem/138624

Copyright

Copyright by Author(s)

This is an article distributed under the terms of the

Creative Commons Attribution 3.0 Unported (CC BY 3.0)

(https://creativecommons.org/licenses/by/3.0/)

\section{Abstract}

Background. A growing number of studies have shown that long-chain non-coding RNA (IncRNA) plays an important role in the progression of non-small cell lung cancer (NSCLC).

Objectives. To explore the role and potential molecular mechanism of IncRNA PSMA3-AS1 in promoting the proliferation, migration and invasion of NSCLC.

Materials and methods. The expression of PSMA3-AS1, miR-17-5p and PD-L1 in a human bronchial epithelial cell line, BEAS-2B, and NSCLC cell lines, H226 and A549, were detected with quantitative real-time polymerase chain reaction ( $Q R T-P C R$ ) or western blot. The PSMA3-AS1 shRNA transfection was used to reduce the expression of PSMA3-AS1. Double fluorescent enzyme reporting was used to detect the relationship between PSMA3-AS1, miR-17-5p and PD-L1. Cell Counting Kit-8 (CCK-8), wound-healing and transwell assays, as well as western blot, were used to detect the expression of proliferation, migration, invasion, and epithelial-mesenchymal transition (EMT)-related proteins in lung cancer cells.

Results. The expression of PSMA3-AS1 in NSCLC cells was significantly higher than in human bronchial epithelial cells. The PSMA3-AS1 knockdown significantly reduced the proliferation, migration and invasion of lung cancer cells. In addition, double fluorescent enzyme results showed that PSMA3-AS1 could competitively bind miR-17-5p to PD-L1. The expression of miR-17-5p is low in lung cancer cells, while the expression of PD-L1 in them is high. Overexpression of PD-L1 reversed the inhibitory effect of PSMA3-AS1 knockdown on the proliferation, migration and invasion of lung cancer cells.

Conclusions. Generally speaking, PSMA3-AS1 is highly expressed in NSCLC. The PSMA3-AS1 can promote the proliferation, migration and invasion of NSCLC cells by regulating miR-17-5p/PD-L1.

Key words: non-small cell lung cancer, proliferation, miR-17-5p, invasion, IncRNA PSMA3-AS1 


\section{Background}

Non-small cell lung cancer (NSCLC) is a common pathological type of lung cancer, accounting for about $80 \%$ of all cases. ${ }^{1}$ At present, the cause of NSCLC is often not completely clear and, due to concealment of NSCLC diagnoses by patients and atypical early clinical features, the early diagnosis rate of NSCLC is low and most patients have metastasis with poor overall prognosis. ${ }^{2}$ Therefore, further in-depth study of lung cancer is of great importance for treating lung cancer and improving the prognosis of patients.

Long chain non-coding RNA (lncRNA) is a non-coding RNA with a length of more than $200 \mathrm{bp}$ that does not encode a protein. It can regulate gene expression through complex molecular mechanisms at many cellular levels, but the specifics of the mechanisms are not clear. ${ }^{3}$ Recent studies have shown that IncRNA, as a regulatory factor, participates in almost all cellular processes and plays a role in promoting or inhibiting cancer. ${ }^{4,5}$ For example, high levels of expression of lncRNA PART1 was found to significantly accelerate the occurrence of NSCLC cancer. ${ }^{6}$ The lncRNA BRCAT54 overexpression promoted proliferation and migration, and activated apoptosis in NSCLC cells by binding RPS9. The lncRNA PSMA3-AS1 is located on chromosome 14 at position $14 \mathrm{q} 23.1$ and is overexpressed in esophageal cancer and glioma. ${ }^{8,9}$ However, the study of PSMA3-AS1 in NSCLC is currently less pressing.

MicroRNAs (miRNAs) are a type of endogenous noncoding RNA with 19-25 nucleotides. They affect cell proliferation, differentiation, migration, and apoptosis by inhibiting the mRNA level of their target gene. ${ }^{10} \mathrm{Mi}$ croRNA is widely reported to be abnormally expressed in NSCLC. ${ }^{11}$ Bioinformatic analysis showed that PSMA3AS1 could target and bind miR-17-5p, and that the expression level of miR-17-5p decreased significantly in lung cancer. ${ }^{12}$ Therefore, we speculate that PSMA3-AS1 can affect the progression of NSCLC by targeting miR-17-5p.

\section{Objectives}

This study aimed to primarily analyze whether PSMA3AS1 targets miR-17-5p and affects biological processes in NSCLC cells, which could provide a new theoretical basis for clinical targeted therapy in NSCLC.

\section{Materials and methods}

\section{Cell culture}

BEAS-2B cells and NSCLC cell lines (H226 and A549, respectively; Shanghai Cell Bank of Chinese Academy of Sciences, Shanghai, China) were cultured in Dulbecco's modified Eagle's medium (DMEM; Thermo Fisher Scientific,
Waltham, USA) containing 10\% fetal bovine serum (FBS; Gibco, Waltham, USA), and subcultured in $5 \% \mathrm{CO}_{2}$ and $37^{\circ} \mathrm{C}$ incubator. Follow-up experiments were carried out on the cells in the logarithmic phase.

\section{Cell transfection}

The shRNA targeting PSMA3-AS1 (sh-PSMA3-AS1), shRNA negative control (sh-NC), the PSMA3-AS1 overexpression plasmid (pcDNA-PSMA3-AS1), the negative control (pcDNA-NC), miR-17-5p mimic, mimic negative control (miR-NC), the PD-L1 overexpression plasmid (OE-PD-L1), and the negative control (OE-NC) were provided from the GenePharma Co., Ltd (Shanghai, China). The Lipofectamine 2000 transfection reagent (Invitrogen, Waltham, USA) was used to transfect the above plasmids into A549 cells.

\section{qRT-PCR analysis}

Total RNA was extracted from cells and synthesized into the cDNA according to a first strand cDNA synthesis kit (Roche Diagnostics, Basel, Switzerland). Quantitative realtime polymerase chain reaction (qRT-PCR) was performed according to the SYBR Green Fast qPCR Mix protocol (Beyotime, Shanghai, China). The $2^{-\Delta \Delta \mathrm{Ct}}$ method was used to analyze the expression of PSMA3-AS1 and miR-17-5p.

\section{CCK-8 assay}

Cells were digested with trypsin and inoculated in 96well plates at a density of $5 \times 10^{5}$ cells $/ \mathrm{mL}$. Routine culture proceeded for $12 \mathrm{~h}$. At the end of culture, Cell Counting Kit-8 (CCK-8) solution ( $10 \mu \mathrm{L}$; Beyotime) was added to each hole of the plates at $24 \mathrm{~h}, 48 \mathrm{~h}$ and $72 \mathrm{~h}$. After $4 \mathrm{~h}$ of culture, the absorbance of each hole at $450 \mathrm{~nm}$ was detected using an automatic enzyme labeling instrument (Thermo Fisher Scientific).

\section{Wound-healing assay}

Cells were inoculated in 12-well plates at a density of $5 \times 10^{4}$ cells $/ \mathrm{mL}$. When the degree of cell fusion was more than $95 \%$, a sterile $200-\mu \mathrm{L}$ liquid transfer gun was used to cross the surface of the culture plate vertically. After $24 \mathrm{~h}$ of routine culture, the scratches were photographed using an optical microscope (Eclipse 80i; Nikon Corp., Tokyo, Japan).

\section{Transwell assay}

Cells were inoculated in the upper chamber (Corning, Inc., Corning, USA) and smeared with Matrigel glue (Becton Dickinson Biosciences, Franklin Lakes, USA) at a density of $5 \times 10^{5}$ cells $/ \mathrm{mL}$; the lower chamber contained $500 \mu \mathrm{L}$ of DMEM complete medium. After $24 \mathrm{~h}$ of routine 


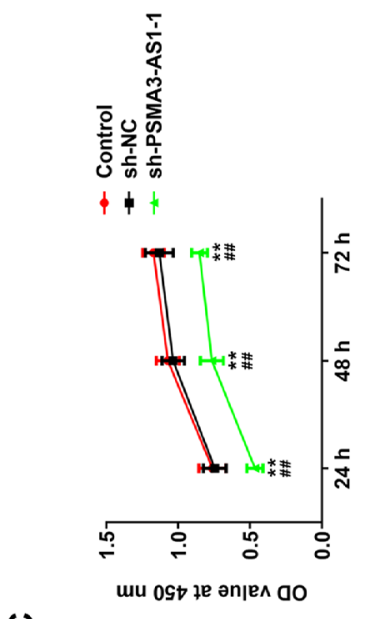

0

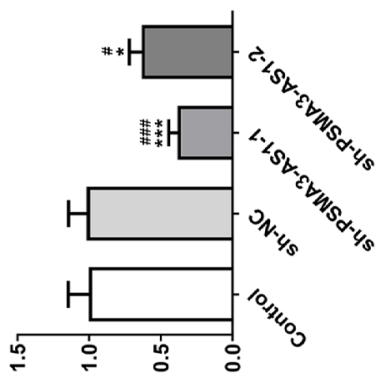

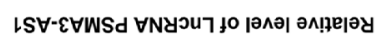

$\infty$

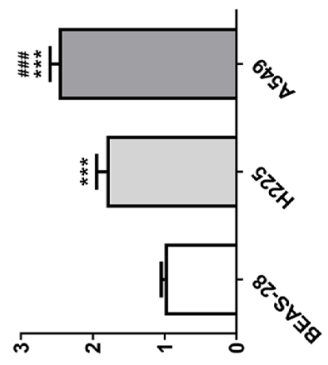

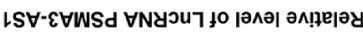

$\varangle$

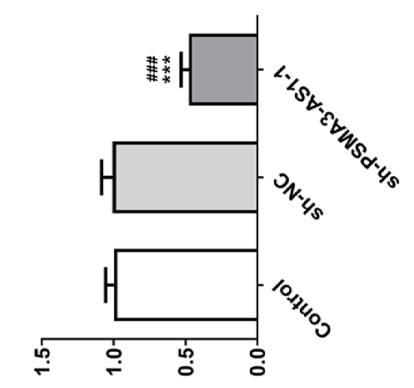

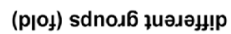

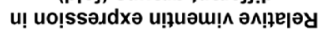

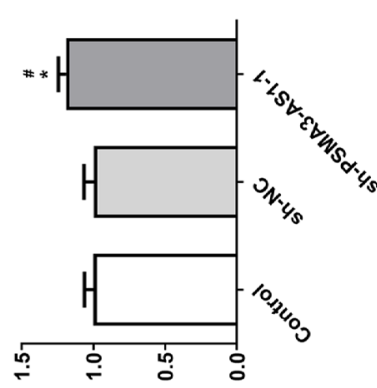

(р|0) sdnoג6 ұนәлән!p

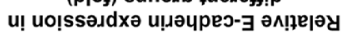

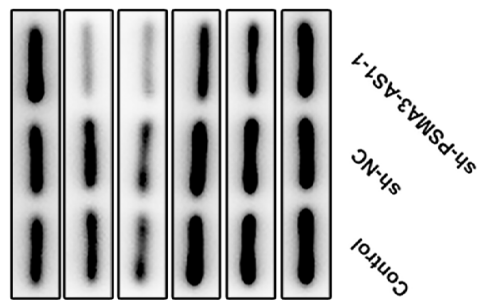

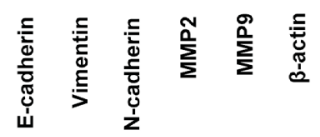

レ

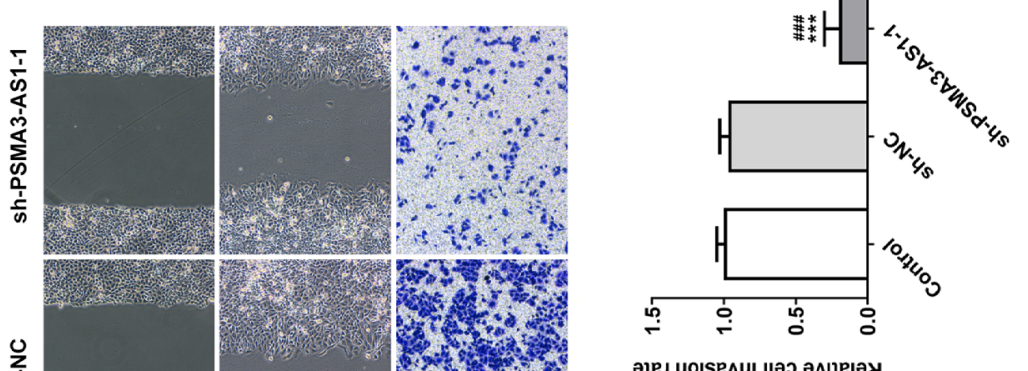

몸

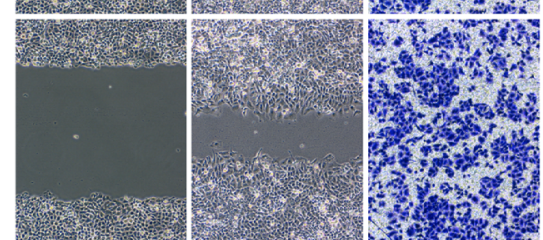

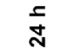

口

uo!̣eม6!̣

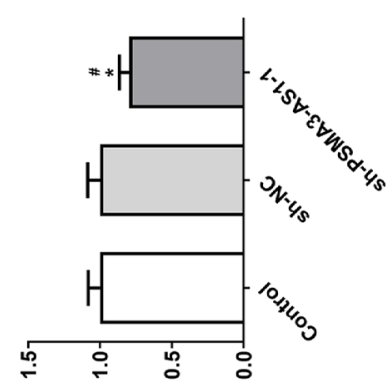

(р|0\}) sdnoג6 ұนәرәщ!p u! uo!ssəudxə 6dWW әм!!e|ə⿳亠口冋
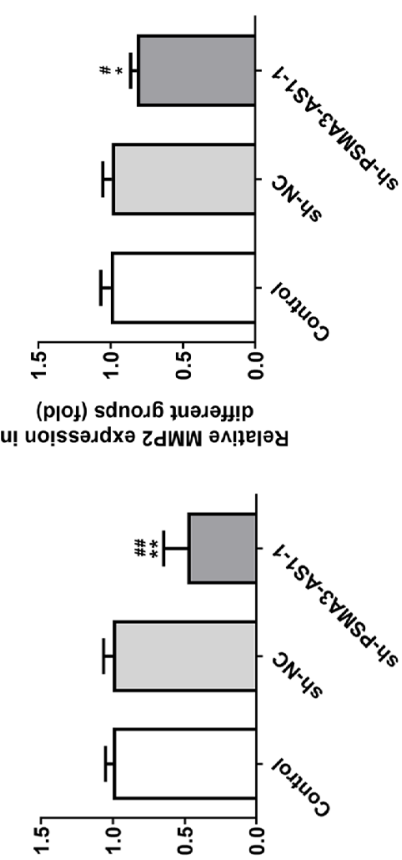

(p/0) sdnod6 ұนәวән!p

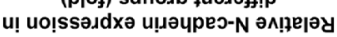

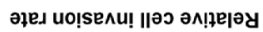

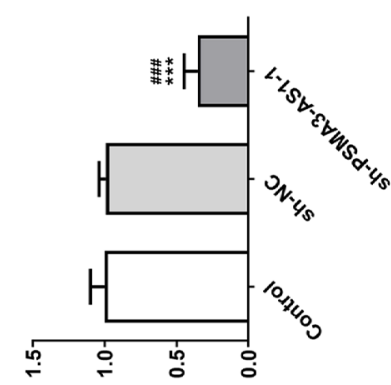

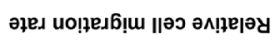

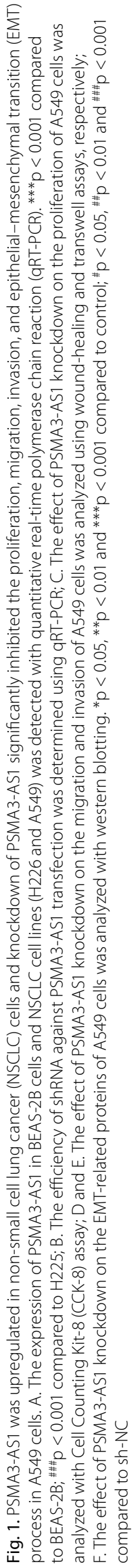


culture, the cells were washed with phosphate-buffered saline (PBS) twice and fixed with paraformaldehyde for $20 \mathrm{~min}$. Then, the uninvaded cells in the upper chamber were gently wiped with a cotton swab. After crystal violet staining for $5 \mathrm{~min}$, the number of invaded cells in each group was observed and counted using a light microscope (Eclipse 80i; Nikon).

\section{Western blot analysis}

Total cellular proteins were extracted from RIPA cell lysate (Beyotime), and the concentration was determined using BCA kit (Bio-Rad, Hercules, USA). The sodium dodecyl sulfate polyacrylamide gel electrophoresis (SDS-PAGE) gel electrophoresis was used to separate the proteins which were transferred to polyvinylidene difluoride (PVDF) membrane. Membranes were sealed with 5\% skim milk at room temperature, incubated with 1:1000 primary antibodies E-cadherin (ab15148; Abcam, Cambridge, UK), N-cadherin (ab12221; Abcam), vimentin (ab45939; Abcam), matrix metalloproteinase (MMP)-2 (ab92536; Abcam), MMP-9 (ab137867; Abcam), and $\beta$-actin (ab8226; Abcam) at $4^{\circ} \mathrm{C}$ for overnight, and then incubated with 1:4000 second antibody anti-rabbit horseradish peroxidase (HRP)-conjugated IgG for another $2 \mathrm{~h}$. The immune bands were visualized using enhanced electrochemiluminescence (ECL) photoluminescence solution (Wanleibio Co., Ltd., Shenyang, China).

\section{Luciferase reporter assay}

Wild-type (WT) and mutant (MUT) reporter plasmids were constructed based on the sequences of PSMA3-
AS1 3'-UTR and the PD-L1 3'-UTR. PSMA3-AS1-WT/Mut or PD-L1-WT/Mut were co-transfected into HEK293T cells with miR-NC and miR-17-5p mimic, respectively. The luciferase activity in the transfected cells was tested using the dual luciferase assay kit (KenReal, Shanghai, China) after $48 \mathrm{~h}$.

\section{Statistical analyses}

Experimental data are presented as mean \pm standard deviation (SD), and the statistical analyses were conducted using GraphPad Prism v. 8 software (GraphPad Software, San Diego, USA). The comparison of differences between 2 groups was carried out with Student's t-test and between multiple groups with one-way analysis of variance (ANOVA) followed by Tukey's post hoc test. The differences were statistically significant at $\mathrm{p}<0.05$.

\section{Results}

\section{Knockdown of PSMA3-AS1 significantly inhibited the proliferation, migration, invasion, and EMT process in A549 cells}

Compared with normal cells, PSMA3-AS1 was upregulated in NSCLC cells, with its highest level of expression in A549 cells (Fig. 1A). Therefore, A549 cells were used for the follow-up study. Subsequently, PSMA3-AS1 was found to be decreased in sh-PSMA3-AS1-1 and -2 groups and the expression of PSMA3-AS1 in the sh-PSMA3-AS1-1 group was the lowest (Fig. 1B). Knockdown of PSMA3-AS1

A

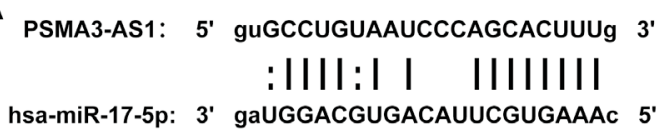

B

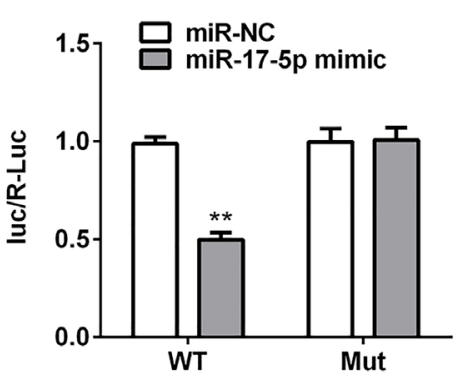

C

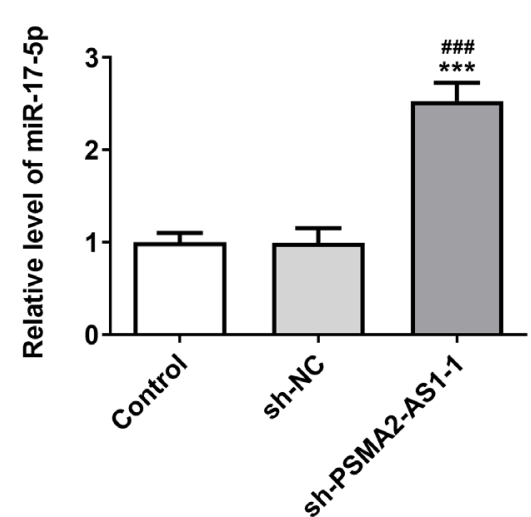

D

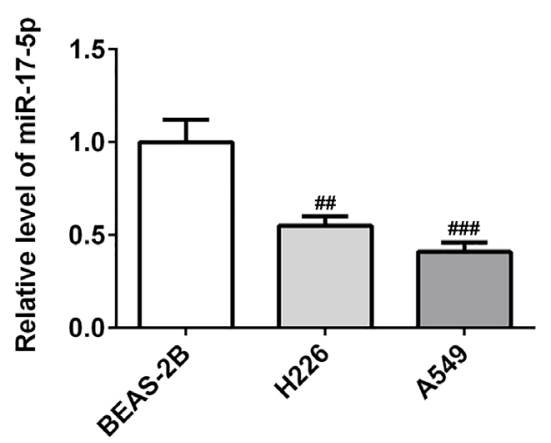

Fig. 2. PSMA3-AS1 negatively regulated the expression of miR-17-5p. A. The binding sites between PSMA3-AS1 and miR-17-5p were predicted using starBase (http://starbase.sysu.edu.cn/); B. The relationship between PSMA3-AS1 and miR-17-5p was determined with luciferase reporter assay. **p $<0.001$ compared to miR-NC; $C$. The effect of PSMA3-AS1 knockdown on the expression of miR-17-5p was detected using quantitative real-time polymerase chain reaction (qRT$P C R)$. ${ }^{* * *} p<0.001$ compared to control; \#\#\# $p<0.001$ compared to sh-NC; D. The expression of miR-17-5p in BEAS-2B cells and non-small cell lung cancer (NSCLC) cell lines (H226 and A549) was detected using qRT-PCR. ${ }^{\# \#} p<0.01$ and ${ }^{\# \# \#} p<0.001$ compared to BEAS-2B 
A

PD-L1: $\quad 5$ ' ...UguuggauuuguaAggcacuuUa... 3 3' $\quad$ C IIIII IIIIIII hsa-miR-17-5p: 3' GAUGGACGUGACAUU--CGUGAAAC 5'

B

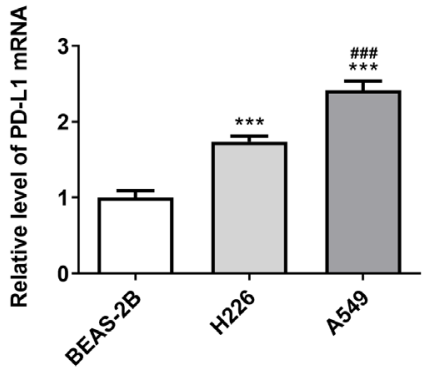

D

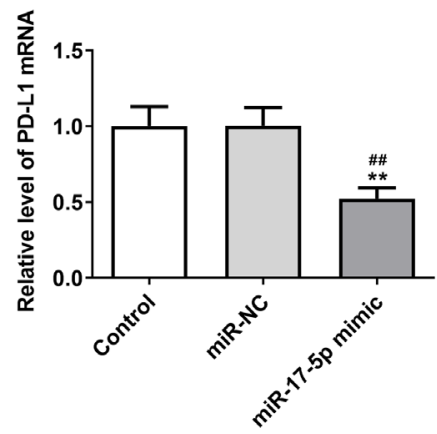

significantly inhibited the activity of A549 cells (Fig. 1C). As shown in Fig. 1D and E, knockdown of PSMA3-AS1 significantly suppressed migration and invasion of A549 cells. In addition, knockdown of PSMA3-AS1 significantly decreased the expression of MMP-2, MMP-9, vimentin, and $\mathrm{N}$-cadherin, whereas it increased the expression of E-cadherin (Fig. 1F).

\section{PSMA3-AS1 negatively regulated miR-17-5p expression}

The miR-17-5p and PSMA3-AS1 binding sites were determined (Fig. 2A). Relative luciferase activity in the PSMA3AS1WT+miR-17-5p mimic group was significantly decreased, but there was no change in the Mut group (Fig. 2B). miR-17-5p was downregulated in NSCLC cells and knockdown of PSMA3-AS1 significantly enhanced the expression level of miR-17-5p (Fig. 2C,D).

\section{miR-17-5p negatively regulated the expression of PD-L1}

The sites of miR-17-5p and PD-L1 binding are indicated in Fig. 3A. PD-L1 was highly expressed in NSCLC cells (Fig. 3B). Upregulation of miR-17-5p significantly reduced the expression of PD-L1 in A549 cells (Fig. 3C,D).
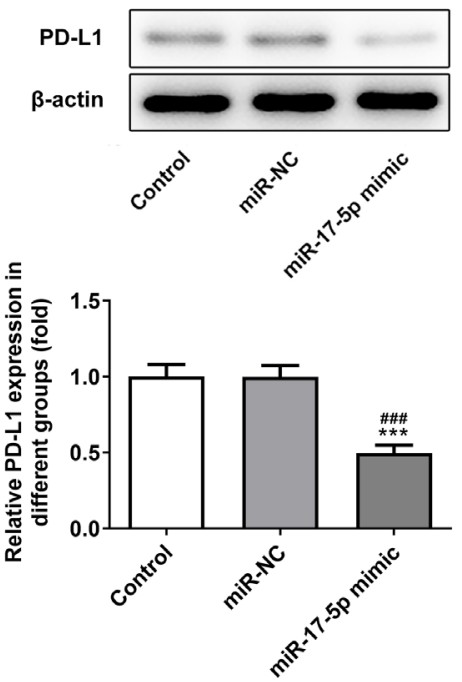

Fig. 3. miR-17-5p negatively regulated the expression of PD-L1. A. The binding sites between miR-17-5p and PD-L1 were predicted using TargetScan database (www.targetscan.org); B. The expression of PD-L1 in BEAS-2B cells and non-small cell lung cancer (NSCLC) cell lines (H226 and A549) was detected using quantitative real-time polymerase chain reaction (qRT-PCR). ${ }^{* *} p<0.001$ compared to BEAS-2B; \#\#\# $\mathrm{p} 0.001$ compared to $\mathrm{H} 225$; C. The effect of miR-17-5p overexpression on the expression of PD-L1 was detected with western blotting; D. The effect of miR-17-5p overexpression on the expression of PD-L1 was detected using qRT-PCR. ${ }^{* *} p<0.01$ and ${ }^{* * *} p<0.001$ compared to control; $\#$ $p<0.01$ and $\# \# \#<0.001$ compared to miR-NC

$\mathrm{NC}$ - negative control.

\section{PSMA3-AS1 regulated the expression of PD-L1 competitively with miR-17-5p}

Relative luciferase activity in the PD-L1 WT+ miR17-5p mimic group was decreased, while it was increased in the PD-L1 WT+ miR-17-5p mimic+PCDNA-PSMA3AS1 group; this indicated that PSMA3-AS1 and PD-L1 could bind to miR-17-5p competitively (Fig. 4A). Upregulation of PSMA3-AS1 significantly promoted the expression of PD-L1, while miR-17-5p overexpression alleviated the enhanced PD-L1 expression caused by overexpression of PSMA3-AS1 (Fig. 4B,C).

\section{PD-L1 reversed the inhibitory effect of PSMA3-AS1 on proliferation, invasion, migration, and the EMT process in A549 cells}

The expression of PD-L1 was increased by transfection with pcDNA-PD-L1 into A549 cells (Fig. 5A). Upregulation of PD-L1 significantly reversed the inhibitory effect of PSMA3-AS1 knockdown on cell activity, migration and invasion (Fig. 5B-D). In addition, compared with the sh-PSMA3-AS1-1 group, upregulation of PD-L1 significantly promoted the expression of vimentin, $\mathrm{N}$-cadherin, MMP-2, and MMP-9, whereas it decreased the expression of E-cadherin (Fig. 5E). 

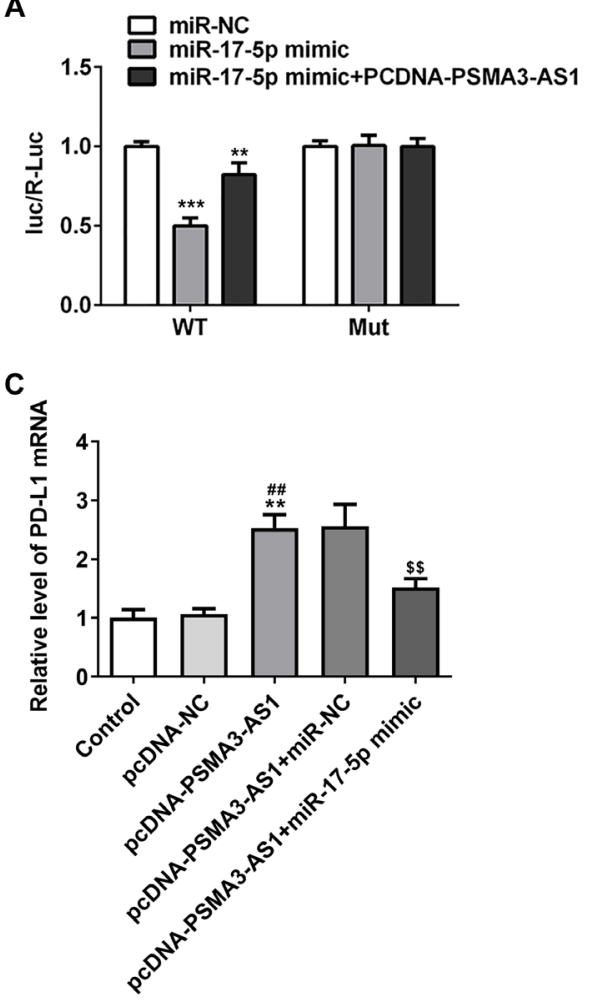

B

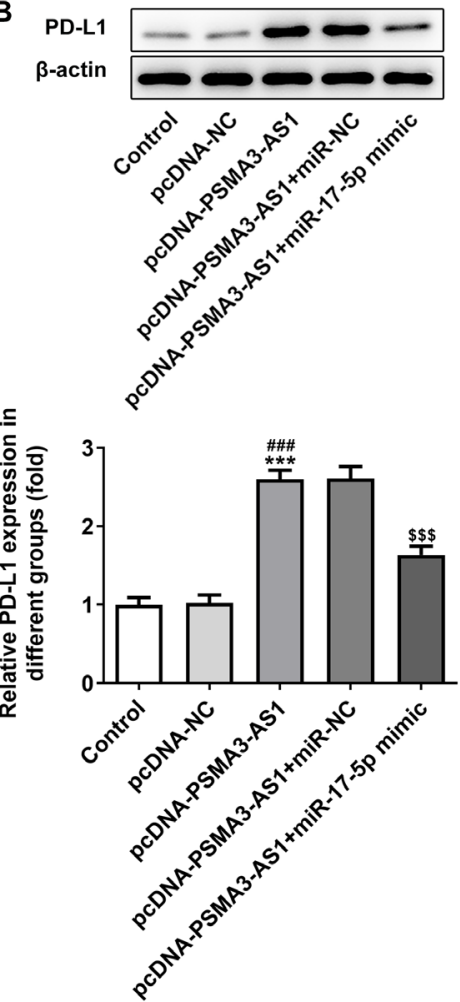

Fig. 4. PSMA3-AS1 regulated the expression of PDL1 through competition combined with miR-17-5p. A. The interaction among PSMA3-AS1, miR-17-5p and PD-L1 was detected using luciferase reporter assay. ${ }^{* *} p<0.01$ and ${ }^{* * *} p<0.001$ compared to miR-NC; B. Effect of miR-17-5p overexpression together with overexpression of PSMA3-AS1 on the expression of PD-L1 was detected with western blotting; C. Effect of miR-17-5p overexpression together with overexpression of PSMA3-AS1 on the expression of PD-L1 was detected using quantitative real-time polymerase chain reaction (qRT-PCR). ${ }^{* *} p<0.01$ and ${ }^{* * *} p<0.001$ compared to control; $* \# p<0.01$ and $\# \#$ $p<0.001$ compared to pcDNA-NC; $\$ p<0.01$ and

${ }^{\$ 5 \$} p<0.001$ compared to pCDNA-PSMA3-AS1+miR-NC WT - wild-type; MUT - mutant; NC - negative control.

\section{Discussion}

The NSCLC is a common respiratory malignancy in adults and is highly susceptible to metastasis. ${ }^{13}$ Therefore, investigating the potential causes and pathogenesis of NSCLC is critical for improving the clinical curative effects of treatments in NSCLC patients.

Many studies have indicated that lncRNAs are involved in the development of NSCLC. ${ }^{14,15}$ Previous studies have shown that PSMA3-AS1 is highly expressed in the majority of tumors. ${ }^{8,9}$ Our results confirmed the high expression of PSMA3-AS1 in NSCLC cells. Knockdown of PSMA3AS1 significantly inhibited the proliferation, migration and invasion of A549 cells. Therefore, upregulation of PSMA3AS1 in NSCLC may play a role in promoting cancer, but its specific regulatory mechanism remains to be studied.

The lncRNAs not only participate in the activation of transcription and the regulation of the expression of surrounding protein-coding genes but are also relevant to many diseases. ${ }^{16,17}$ In addition, lncRNA could affect the role of relevant functional proteins through the adsorption of miRNA. ${ }^{18,19}$ For example, the lncRNA SNHG15 accelerates the progression of lung cancer cells by targeting miR-211-3p. ${ }^{20}$ The lncRNA PVT1-5 facilitates metastasis of lung cancer cells by suppressing the expression of miR126. ${ }^{21} \mathrm{MiR}-17-5 p$ was selected as a target gene of PSMA3AS1 by screening the starBase (http://starbase.sysu.edu. $\mathrm{cn} /$ ) database. In thyroid cancer, miR-17-5p aggravates proliferation and invasiveness of thyroid cancer cells. ${ }^{22}$ In triple-negative breast cancer (TNBC), $m i R-17-5 p$ can suppress the occurrence of TNBC. ${ }^{23}$ In this study, $m i R-17-5 p$ was downregulated in NSCLC and $m i R-17-5 p$ overexpression significantly reduced the facilitation effect of PSMA3-AS1 on the proliferation, migration and invasion of A 549 cells.

For NSCLC treatment, PD-L1 inhibitors could effectively block T-cell proliferation and improve survival of NSCLC patients. ${ }^{24,25}$ Meanwhile, PD-L1 was highly expressed in the serum of patients with advanced lung cancer, suggesting that a high level of PD-L1 was correlated with a worse outcome. ${ }^{26}$ In the present study, we found that miR-17-5p could bind to PD-L1. PD-L1 was upregulated in NSCLC, and the effect of PSM3-AS1 downregulation on cancer progression could be reversed by the upregulation of PD-L1.

\section{Limitations}

In our study, we only carried out cellular experiments to investigate the role of PSMA3-AS1 in NSCLC progression by targeting miR-17-5p/PD-L1. However, determining the effect of PSMA3-AS1 inhibition on NSCLC progression in animal experiments and clinical scenarios, as well as the exact mechanism underlying the effect, requires further research.

\section{Conclusions}

PSMA3-AS1 was highly expressed in NSCLC. PSMA3AS1 promoted the proliferation, migration and invasion of NSCLC through upregulation of PD-L1 by targeting miR-17-5p. However, the present study is based on cellular 
A

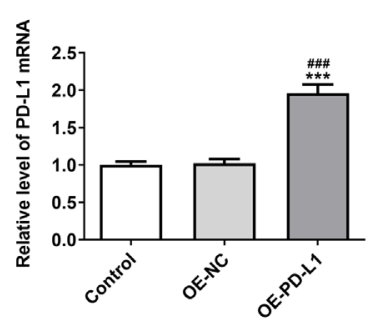

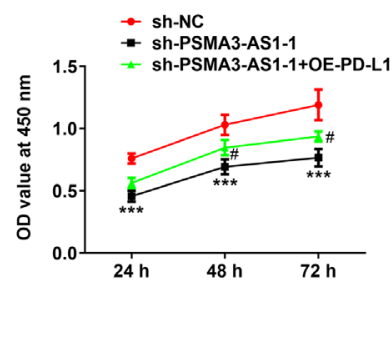

C

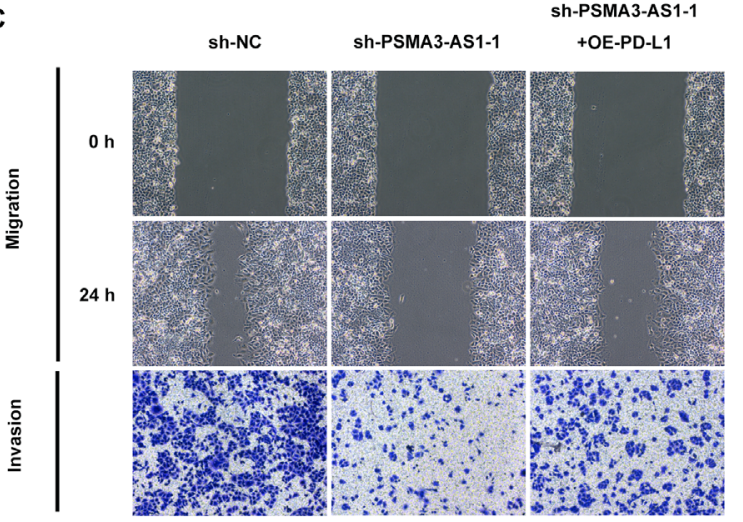

E
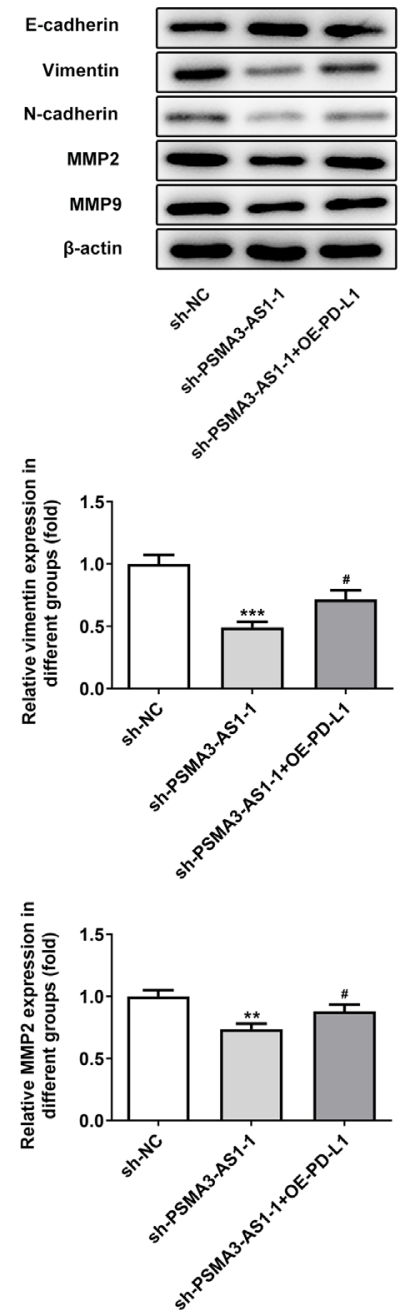
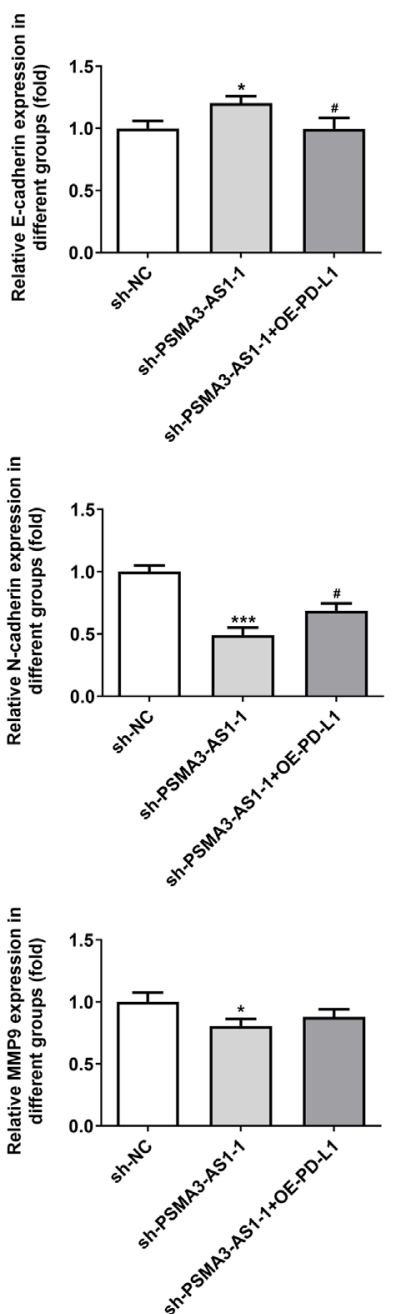

Fig. 5. PD-L1 reversed the inhibitory effect of PSMA3-AS1 on proliferation, invasion, migration, and epithelial-mesenchymal transition (EMT) process in A549 cells. A. The efficiency of PD-L1 overexpression was examined using quantitative real-time polymerase chain reaction (qRT-PCR). ${ }^{* * *} p<0.001$ compared to control; \#\#\# < 0.001 compared to OE-NC; B. Effect of PSM3-AS1 knockdown together with PD-L1 overexpression on the proliferation of A549 cells was analyzed using Cell Counting Kit-8 (CCK-8) assay; D and E. Effect of PSMA3-AS1 knockdown together with PD-L1 overexpression on the migration and invasion of A549 cells was analyzed using wound-healing and transwell assays, respectively; F. Effect of PSMA3-AS1 knockdown together with PD-L1 overexpression on the EMT-related proteins (E-cadherin, vimentin, N-cadherin, matrix metalloproteinase-2 (MMP-2), and MMP-9) of A549 cells was analyzed with western blotting. ${ }^{*} \mathrm{p}<0.05,{ }^{* *} \mathrm{p}<0.01$ and ${ }^{* * *} \mathrm{p}<0.001$ compared to sh-NC; ${ }^{*} \mathrm{p}<0.05$, ${ }^{* \#} \mathrm{p}<0.01$ compared to sh-PSMA3-AS1-1

NC - negative control; sh - sham.

experiments; thus, the detection of PSMA3-AS1 and miR17-5p in NSCLC tissues and verification with animal experiments will be important to accomplish in future studies.

\section{ORCID iDs}

Guohua Cheng (1) https://orcid.org/0000-0001-9751-0792 Yarong Li (10) https://orcid.org/0000-0002-3572-002X Zhaoyu Liu (1) https://orcid.org/0000-0001-7104-1393 Xiang Song (1) https://orcid.org/0000-0003-2138-0463

\section{References}

1. Cao M, Chen W. Epidemiology of lung cancer in China. Thorac Cancer. 2019;10(1):3-7. doi:10.1111/1759-7714.12916

2. Li M, Chen R, Ji B, et al. RAD52 variants influence NSCLC risk in the Chinese population in a high altitude area. Ther Adv Respir Dis. 2020;14: 1753466620918192. doi:10.1177/1753466620918192

3. Feng C, Zhao Y, LiY,Zhang T, Ma Y, Liu Y.LncRNA MALAT1 promotes lung cancer proliferation and gefitinib resistance by acting as a miR-200a sponge. Arch Bronconeumol. 2019;55(12):627-633. doi:10.1016/j. arbres.2019.03.026
4. Zhou Y, Shi H, Du Y, et al. IncRNA DLEU2 modulates cell proliferation and invasion of non-small cell lung cancer by regulating miR-30c-5p/SOX9 axis.Aging (Albany NY). 2019;11(18):7386-7401.doi:10.18632/aging.102226

5. Huang N, Guo W, Ren K, et al. LncRNA AFAP1-AS1 supresses miR-139$5 p$ and promotes cell proliferation and chemotherapy resistance of non-small cell lung cancer by competitively upregulating RRM2. Front Oncol. 2019;9:1103. doi:10.3389/fonc.2019.01103

6. Chen Y, Zhou X, Huang C, et al. LnCRNA PART1 promotes cell proliferation and progression in non-small-cell lung cancer cells via sponging miR-17-5p.J Cell Biochem. 2021;122(3-4):315-325. doi:10.1002/jcb.29714

7. Yang W, Qian Y, Gao K, et al. LncRNA BRCAT54 inhibits the tumorigenesis of non-small cell lung cancer by binding to RPS9 to transcriptionally regulate JAK-STAT and calcium pathway genes. Carcinogenesis. 2021;42(1):80-92. doi:10.1093/carcin/bgaa051

8. Qiu BQ, Lin XH, Ye XD, et al. Long non-coding RNA PSMA3-AS1 promotes malignant phenotypes of esophageal cancer by modulating the miR-101/EZH2 axis as a ceRNA. Aging (Albany NY). 2020;12(2): 1843-1856. doi:10.18632/aging.102716

9. Zhou LL, Zhang M, Zhang YZ, Sun MF. Long non-coding RNA PSMA3AS1 enhances cell proliferation, migration and invasion by regulating miR-302a-3p/RAB22A in glioma. Biosci Rep. 2020;40(9):BSR20191571. doi:10.1042/bsr20191571 
10. Giallombardo M, Chacártegui Borrás J, Castiglia M, et al. Exosomal miRNA analysis in non-small cell lung cancer (NSCLC) patients' plasma through qPCR: A feasible liquid biopsy tool. J Vis Exp. 2016;111: 53900. doi:10.3791/53900

11. Jin X, Guan $\mathrm{Y}$, Zhang Z, Wang $\mathrm{H}$. Microarray data analysis on gene and miRNA expression to identify biomarkers in non-small cell lung cancer. BMC Cancer. 2020;20(1):329. doi:10.1186/s12885-020-06829-X

12. Xu C, Zheng Y, Lian D, Ye S, Yang J, Zeng Z. Analysis of microRNA expression profile identifies novel biomarkers for non-small cell lung cancer. Tumori. 2015;101(1):104-110. doi:10.5301/tj.5000224

13. Duma N, Santana-Davila R, Molina JR. Non-small cell lung cancer: Epidemiology, screening, diagnosis, and treatment. Mayo Clin Proc. 2019;94(8):1623-1640. doi:10.1016/j.mayocp.2019.01.013

14. Yang H, Yang W, Dai W, Ma Y, Zhang G. LINC00667 promotes the proliferation, migration, and pathological angiogenesis in non-small cell lung cancer through stabilizing VEGFA by EIF4A3. Cell Biol Int. 2020;44(8):1671-1680. doi:10.1002/cbin.11361

15. Yan Z, Yang $Q$, Xue $M$, Wang $S$, Hong W, Gao X. YY1-induced IncRNA ZFPM2-AS1 facilitates cell proliferation and invasion in small cell lung cancer via upregulating of TRAF4. Cancer Cell Int. 2020;20:108. doi:10.1186/s12935-020-1157-7

16. Zhou S, Wang L, Yang Q, et al. Systematical analysis of IncRNA-mRNA competing endogenous RNA network in breast cancer subtypes. Breast Cancer Res Treat. 2018;169(2):267-275. doi:10.1007/s10549-0184678-1

17. Zhao $\mathrm{Y}$, Wang $\mathrm{H}, \mathrm{Wu} \mathrm{C}$, et al. Construction and investigation of IncRNAassociated ceRNA regulatory network in papillary thyroid cancer. Oncol Rep. 2018;39(3):1197-1206. doi:10.3892/or.2018.6207

18. Ma L, Sun X, Kuai W, et al. LncRNA SOX2 overlapping transcript acts as a miRNA sponge to promote the proliferation and invasion of Ewing's sarcoma. Am J Transl Res. 2019;11(6):3841-3849. PMID:31312393
19. D'Angelo D, Mussnich P, Sepe R, et al. RPSAP52 IncRNA is overexpressed in pituitary tumors and promotes cell proliferation by acting as miRNA sponge for HMGA proteins. J Mol Med (Berl). 2019;97(7): 1019-1032. doi:10.1007/s00109-019-01789-7

20. Cui HX, Zhang MY, Liu K, Liu J, Zhang ZL, Fu L. LncRNA SNHG15 promotes proliferation and migration of lung cancer via targeting microRNA211-3p. Eur Rev Med Pharmacol Sci. 2018;22(20):6838-6844. doi:10. 26355/eurrev_201810_16152

21. Li H, Chen S, Liu J, et al. Long non-coding RNA PVT1-5 promotes cell proliferation by regulating miR-126/SLC7A5 axis in lung cancer. Biochem Biophys Res Commun. 2018;495(3):2350-2355. doi:10.1016/j. bbrc.2017.12.114

22. Shi YP, Liu GL, Li S, Liu XL. miR-17-5p knockdown inhibits proliferation, autophagy and promotes apoptosis in thyroid cancer via targeting PTEN. Neoplasma. 2020;67(2):249-258. doi:10.4149/neo_2019_ $190110 \mathrm{~N} 29$

23. Li J, Lai Y, Ma J, et al. miR-17-5p suppresses cell proliferation and invasion by targeting ETV1 in triple-negative breast cancer. BMC Cancer. 2017;17(1):745. doi:10.1186/s12885-017-3674-x

24. Bylicki O, Paleiron N, Rousseau-Bussac G, Chouaïd C. New PDL1 inhibitors for non-small cell lung cancer: Focus on pembrolizumab. Onco Targets Ther. 2018;11:4051-4064. doi:10.2147/ott.s154606

25. Dong H, Strome SE, Salomao DR, et al. Tumor-associated B7-H1 promotes T-cell apoptosis: A potential mechanism of immune evasion. Nat Med. 2002;8(8):793-800. doi:10.1038/nm730

26. Okuma Y, Hosomi Y, Nakahara Y, Watanabe K, Sagawa Y, Homma S. High plasma levels of soluble programmed cell death ligand 1 are prognostic for reduced survival in advanced lung cancer. Lung Cancer. 2017;104:1-6. doi:10.1016/j.lungcan.2016.11.023 